LAWRENCE LIVERMORE NATIONAL LABORATORY

\title{
Study of Compton vs. Photoelectric Interactions
}

J. Gronberg, S. Johnson, D. Lange, D. Wright

July 26, 2004 


\section{Disclaimer}

This document was prepared as an account of work sponsored by an agency of the United States Government. Neither the United States Government nor the University of California nor any of their employees, makes any warranty, express or implied, or assumes any legal liability or responsibility for the accuracy, completeness, or usefulness of any information, apparatus, product, or process disclosed, or represents that its use would not infringe privately owned rights. Reference herein to any specific commercial product, process, or service by trade name, trademark, manufacturer, or otherwise, does not necessarily constitute or imply its endorsement, recommendation, or favoring by the United States Government or the University of California. The views and opinions of authors expressed herein do not necessarily state or reflect those of the United States Government or the University of California, and shall not be used for advertising or product endorsement purposes.

\section{Auspices Statement}

This work was performed under the auspices of the U.S. Department of Energy by University of California, Lawrence Livermore National Laboratory under Contract W-7405-Eng-48. 
$\mathrm{h} 4 \mathrm{doc} 2 \mathrm{v} 1$

\title{
Study of Compton vs. Photoelectric Interactions
}

\author{
Jeff Gronberg, Stephen Johnson, David Lange, Doug Wright \\ High Energy Physics Group, N Division
}

September 3, 2003

\section{Introduction}

We have studied how often incoming photons interact via a Compton interaction and/or a photoelectric interaction as a function of energy and detector material Results are using a $1 \mathrm{~m}^{3}$ detector, and discrete energy photons from $0.1 \mathrm{MeV}$ up to $10 \mathrm{MeV}$. Essentially all of the lower energy photons interact at least once in a detector of this size. This is not the case at higher energies. Each detector, photon energy combination was simulated with 2000 photons.

The following pages contain plots and tables showing how the following quantities vary with photon energy and detector material:

1. Fraction of photons that interact via the Compton interaction at least once.

2. Fraction of photons that do not interact via the Compton interaction, but rather are absorbed via a photoelectric interaction.

3. Fraction of photons that interact via the Compton interaction exactly once and are then absorbed via a photoelectric interaction.

4. Fraction of photons that interact via the Compton interaction at least twice.

Table 0: Summary of detector properties. The "Plastic" is polystyene scintillator. The "Glass" is a proprietary mixture used by Arno Ledebuhr from Collimated Holes, Inc. It has a LKH-6 glass core and borosilicate glass cladding.

\begin{tabular}{cccc}
\hline Detector & Radiation length $(\mathrm{cm})$ & Density $\left(\mathrm{g} / \mathrm{cm}^{3}\right)$ & Effective Z \\
\hline \hline Germanium & 2.30 & 5.32 & 32 \\
Glass & 4.48 & 3.27 & $\approx 31$ \\
Silicon & 9.37 & 2.33 & 14 \\
Plastic & 42.55 & 1.03 & $\approx 4$ \\
\hline
\end{tabular}




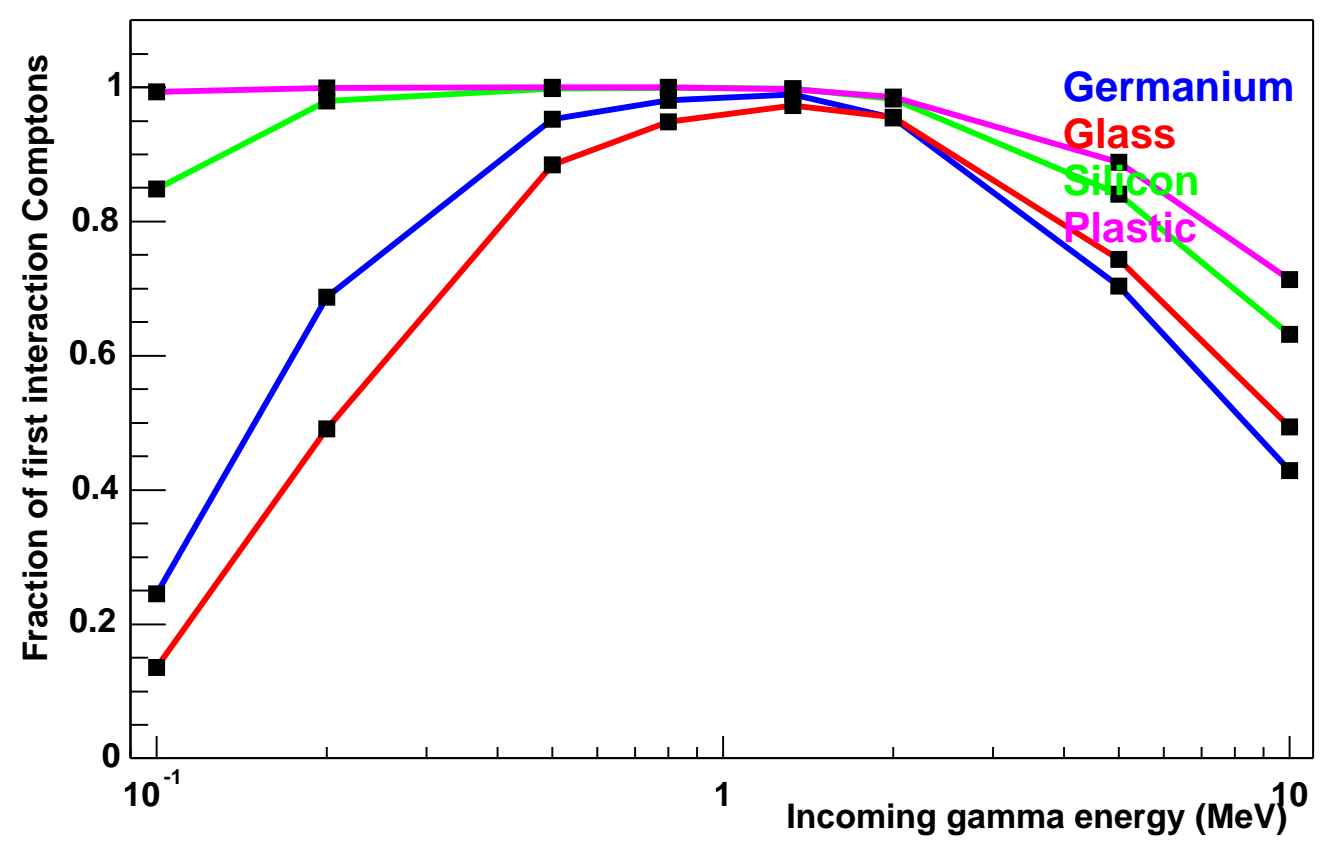

Figure 1: Fraction of gammas that undergo a Compton interaction at least once in a $1 \mathrm{~m}^{3}$ detector. Results are shown for the glass, plastic, silicon, and germanium based detectors.

Table 1: Percentage of gammas that undergo a Compton interaction as their first interaction in a $1 \mathrm{~m}^{3}$ detector.

\begin{tabular}{ccccc}
\hline Energy $(\mathrm{MeV})$ & Germanium & Glass & Silicon & Plastic \\
\hline \hline 0.1 & $24.55 \pm 0.43$ & $13.59 \pm 0.34$ & $84.89 \pm 0.36$ & $99.35 \pm 0.08$ \\
0.2 & $68.74 \pm 0.46$ & $49.11 \pm 0.50$ & $97.98 \pm 0.14$ & $99.90 \pm 0.03$ \\
0.5 & $95.27 \pm 0.21$ & $88.49 \pm 0.32$ & $99.83 \pm 0.04$ & $99.97 \pm 0.02$ \\
0.8 & $98.04 \pm 0.14$ & $94.83 \pm 0.22$ & $99.93 \pm 0.03$ & $99.96 \pm 0.02$ \\
1.33 & $98.96 \pm 0.10$ & $97.30 \pm 0.16$ & $99.82 \pm 0.04$ & $99.71 \pm 0.05$ \\
2 & $95.46 \pm 0.21$ & $95.57 \pm 0.21$ & $98.22 \pm 0.13$ & $98.57 \pm 0.12$ \\
5 & $70.37 \pm 0.46$ & $74.38 \pm 0.44$ & $84.12 \pm 0.37$ & $88.88 \pm 0.31$ \\
10 & $42.90 \pm 0.49$ & $49.44 \pm 0.50$ & $63.17 \pm 0.48$ & $71.30 \pm 0.45$ \\
\hline
\end{tabular}




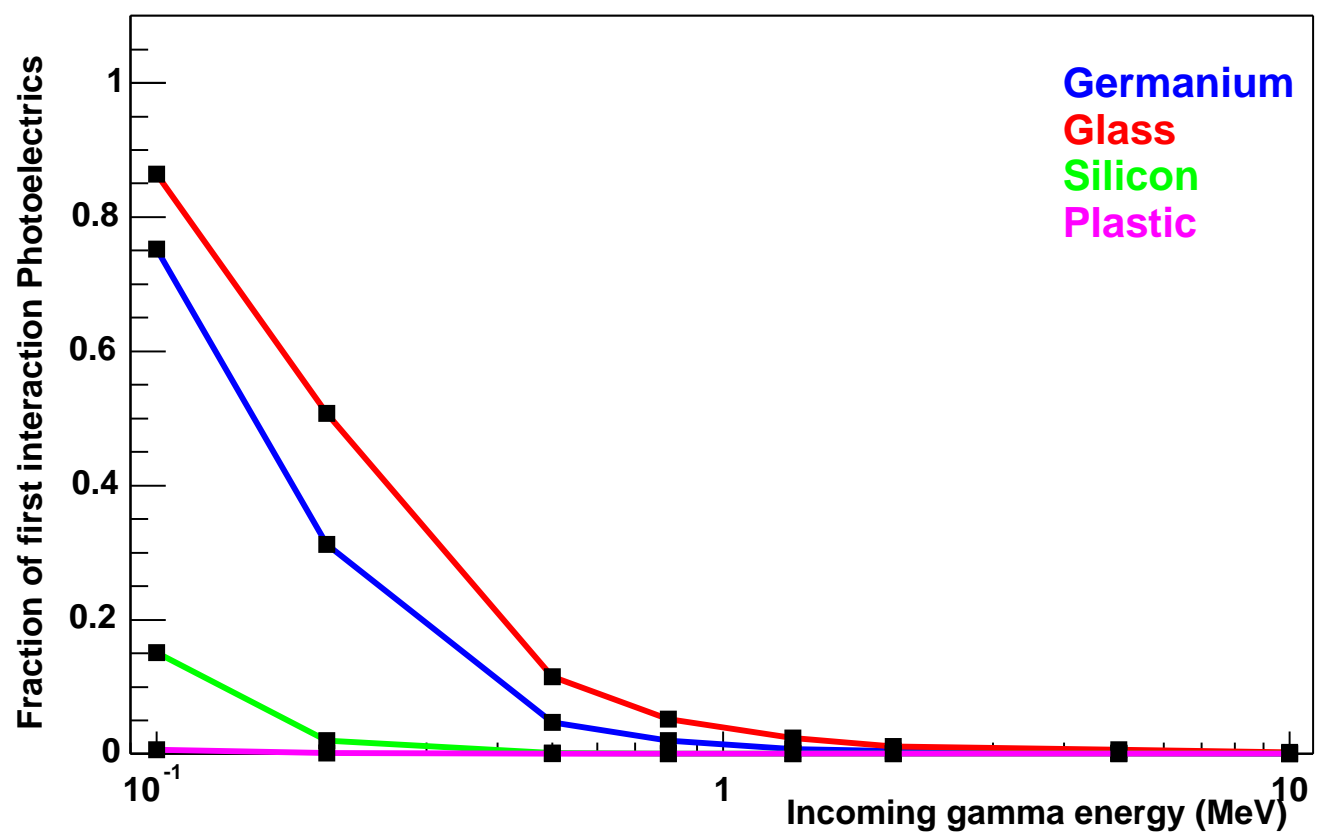

Figure 2: Fraction of gammas that undergo a photoelectric interaction as their first interaction in a $1 \mathrm{~m}^{3}$ detector. Results are shown for the glass, plastic, silicon, and germanium based detectors.

Table 2: Percentage of gammas that undergo a photoelectric interaction as their first interaction in a $1 \mathrm{~m}^{3}$ detector.

\begin{tabular}{ccccc}
\hline Energy $(\mathrm{MeV})$ & Germanium & Glass & Silicon & Plastic \\
\hline \hline 0.1 & $75.3 \pm 0.43$ & $86.4 \pm 0.34$ & $15.1 \pm 0.36$ & $0.7 \pm 0.08$ \\
0.2 & $31.3 \pm 0.46$ & $50.8 \pm 0.50$ & $2.0 \pm 0.14$ & $0.1 \pm 0.03$ \\
0.5 & $4.7 \pm 0.21$ & $11.5 \pm 0.32$ & $0.2 \pm 0.04$ & $0.0 \pm 0.01$ \\
0.8 & $2.0 \pm 0.14$ & $5.2 \pm 0.22$ & $0.1 \pm 0.03$ & $0.0 \pm 0.00$ \\
1.33 & $0.7 \pm 0.08$ & $2.3 \pm 0.15$ & $0.1 \pm 0.02$ & $0.0 \pm 0.00$ \\
2 & $0.5 \pm 0.07$ & $1.1 \pm 0.10$ & $0.1 \pm 0.03$ & $0.0 \pm 0.00$ \\
5 & $0.2 \pm 0.04$ & $0.6 \pm 0.08$ & $0.0 \pm 0.01$ & $0.0 \pm 0.00$ \\
10 & $0.1 \pm 0.03$ & $0.3 \pm 0.05$ & $0.0 \pm 0.01$ & $0.0 \pm 0.00$ \\
\hline
\end{tabular}




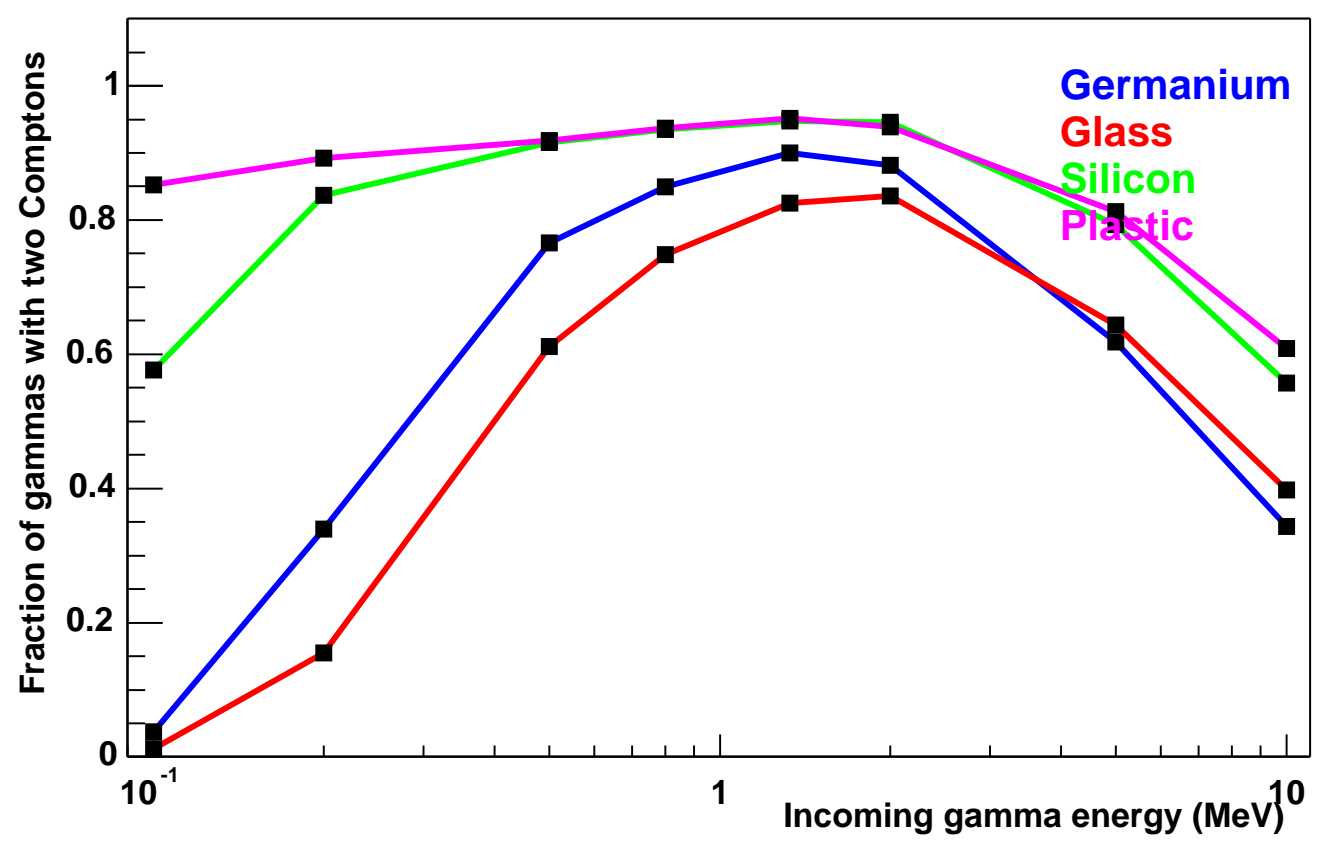

Figure 3: Fraction of gammas that undergo two Compton interactions as their first two interactions in a $1 \mathrm{~m}^{3}$ detector. Results are shown for the glass, plastic, silicon, and germanium based detectors.

Table 3: Percentage of gammas that undergo a two Compton interactions as their first two interactions in a $1 \mathrm{~m}^{3}$ detector.

\begin{tabular}{ccccc}
\hline Energy $(\mathrm{MeV})$ & Germanium & Glass & Silicon & Plastic \\
\hline \hline 0.1 & $3.7 \pm 0.19$ & $1.2 \pm 0.11$ & $57.6 \pm 0.49$ & $85.2 \pm 0.36$ \\
0.2 & $33.9 \pm 0.47$ & $15.5 \pm 0.36$ & $83.7 \pm 0.37$ & $89.3 \pm 0.31$ \\
0.5 & $76.6 \pm 0.42$ & $61.1 \pm 0.49$ & $91.5 \pm 0.28$ & $91.9 \pm 0.27$ \\
0.8 & $84.9 \pm 0.36$ & $74.8 \pm 0.43$ & $93.5 \pm 0.25$ & $93.7 \pm 0.24$ \\
1.33 & $90.0 \pm 0.30$ & $82.5 \pm 0.38$ & $94.8 \pm 0.22$ & $95.1 \pm 0.22$ \\
2 & $88.1 \pm 0.32$ & $83.6 \pm 0.37$ & $94.6 \pm 0.23$ & $93.8 \pm 0.24$ \\
5 & $61.8 \pm 0.49$ & $64.4 \pm 0.48$ & $79.3 \pm 0.41$ & $81.3 \pm 0.39$ \\
10 & $34.3 \pm 0.47$ & $39.8 \pm 0.49$ & $55.7 \pm 0.50$ & $60.8 \pm 0.49$ \\
\hline
\end{tabular}




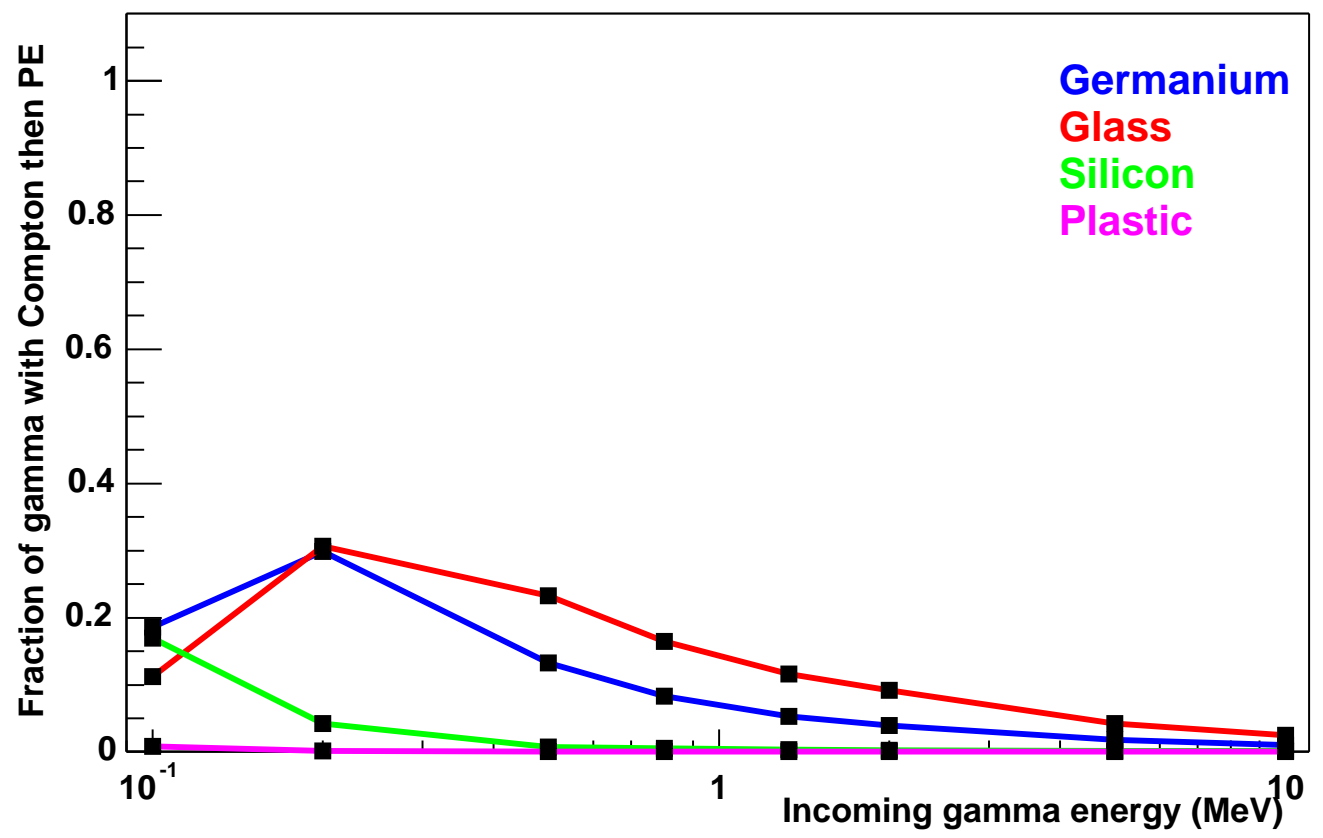

Figure 4: Fraction of gammas that undergo one Compton interaction followed by a photoelectric interaction in a $1 \mathrm{~m}^{3}$ detector. Results are shown for the glass, plastic, silicon, and germanium based detectors.

Table 4: Percentage of gammas that undergo a Compton interaction followed by a photoelectric interaction in a $1 \mathrm{~m}^{3}$ detector.

\begin{tabular}{ccccc}
\hline Energy $(\mathrm{MeV})$ & Germanium & Glass & Silicon & Plastic \\
\hline \hline 0.1 & $18.6 \pm 0.39$ & $11.3 \pm 0.32$ & $17.0 \pm 0.38$ & $0.8 \pm 0.09$ \\
0.2 & $29.9 \pm 0.46$ & $30.6 \pm 0.46$ & $4.2 \pm 0.20$ & $0.2 \pm 0.04$ \\
0.5 & $13.3 \pm 0.34$ & $23.2 \pm 0.42$ & $0.7 \pm 0.09$ & $0.0 \pm 0.02$ \\
0.8 & $8.3 \pm 0.28$ & $16.5 \pm 0.37$ & $0.5 \pm 0.07$ & $0.0 \pm 0.00$ \\
1.33 & $5.2 \pm 0.22$ & $11.6 \pm 0.32$ & $0.3 \pm 0.06$ & $0.0 \pm 0.01$ \\
2 & $4.0 \pm 0.20$ & $9.2 \pm 0.29$ & $0.2 \pm 0.05$ & $0.0 \pm 0.01$ \\
5 & $1.8 \pm 0.13$ & $4.3 \pm 0.20$ & $0.2 \pm 0.04$ & $0.0 \pm 0.01$ \\
10 & $1.0 \pm 0.10$ & $2.5 \pm 0.16$ & $0.1 \pm 0.03$ & $0.0 \pm 0.00$ \\
\hline
\end{tabular}




\section{Appendix}

For reference, we include the PDG plots of photon total cross sections for carbon and lead.

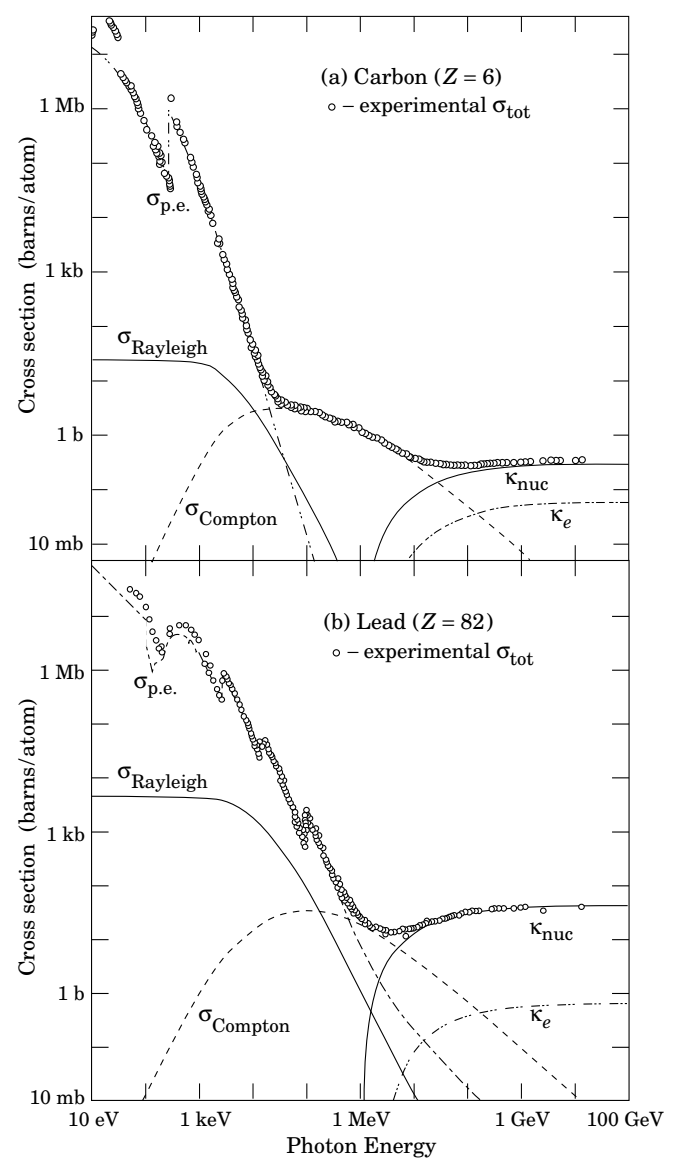

Figure 26.13: Photon total cross sections as a function of energy in carbon and lead, showing the contributions of different processes:

$\sigma_{\text {p.e. }}=$ Atomic photoelectric effect (electron ejection, photon absorption)

$\sigma_{\text {Rayleigh }}=$ Coherent scattering (Rayleigh scattering-atom neither ionized nor excited)

$\sigma_{\text {Compton }}=$ Incoherent scattering (Compton scattering off an electron)

$\kappa_{\text {nuc }}=$ Pair production, nuclear field

$\kappa_{e}=$ Pair production, electron field

Data from Hubbell, Gimm, and Øverbø, J. Phys. Chem. Ref. Data 9, 1023 (1980). Curves for these and other elements, compounds, and mixtures may be obtained from

http://physics.nist.gov/PhysRefData. The photon total cross section is approximately flat for at least two decades beyond the energy range shown. Original figures courtesy J.H. Hubbell (NIST). 